\title{
Topological Superconductivity in a Phase-Controlled Quasi-one-dimensional planar Josephson-Junction
}

\section{Evidence of topological superconductivity in planar Josephson junctions} Authors: Antonio Fornieri, Alexander M. Whiticar, F. Setiawan, Elías Portolés Marín, Asbjørn C. C. Drachmann, Anna Keselman, Sergei Gronin, Candice Thomas, Tian Wang, Ray Kallaher, Geoffrey C. Gardner, Erez Berg, Michael J. Manfra, Ady Stern, Charles M. Marcus, Fabrizio Nichele

Nature 569, 89-92 (2019)

\section{Topological Superconductivity in a Phase-Controlled Josephson Junction} Authors: Hechen Ren, Falko Pientka, Sean Hart, Andrew Pierce, Michael Kosowsky, Lukas Lunczer, Raimund Schlereth, Benedikt Scharf, Ewelina M. Hankiewicz, Laurens W. Molenkamp, Bertrand I. Halperin, Amir Yacoby Nature 569, 93-98 (2019)

\section{Recommended with a Commentary by Yuval Oreg, Department of Condensed Matter Physics, Weizmann Institute of Science, Rehovot, Israel 76100}

\section{Introduction}

In the last decade we have witnessed a tremendous endeavor to find robust Majorana Zero Modes (MZMs) at the edge of quasi-one-dimensional systems exhibiting the spinless $p$-wave topological superconducting state of matter. The motivation for realizing such a phase is the fundamental physics aspiration for discovering novel states of matter, and because MZMs are identified as key ingredients for topological quantum information processing.

Several platforms have been proposed for realizing topological superconductivity. Numerous reviews on the field have been published, including few recommendations and comments on specific progresses here at the journal club [1]. For a recent review which concentrates on the experimental progress in semiconductingsuperconducting systems see Ref. [2]. Stabilization of this sought-after topological superconducting state requires a proper combination of superconductivity and spin rotations that breaks the time-reversal symmetry.

The two theoretical papers by Hell et al. [3] and by Pientka and Keselman et al. [4], hereafter referred to as the HKP theory, were followed by the two experiments at Copenhagen and Harvard, which I recommend here. These two experiments form an important 
progress in the field, as they demonstrate the possibility to use the superconducting phase difference of a planar Josephson junction to manipulate the properties of the hybrid system and tune it to a topological state. The hope is that by controlling the phase difference, that already breaks time-reversal symmetry, we can stabilize the topological superconducting state with a relatively small external magnetic (Zeeman) field, which is less harmful to the superconductor's properties.

Ever since the field's nascent stages, it was theoretically recognized that manipulation of the phase of the superconducting pair potential may help to create Majorana Zero Modes and stabilize quasi-onedimensional topological states. For example, Ref. [5] discusses how super-current along a one-dimensional wire, that causes the superconducting phase to increase linearly along the wire, tunes the system to a topological regime. In a recent remarkable experiment, that I highly recommend, a 1D wire is covered all around by a superconductor forming a full-shell superconductor. Then, a phase winding of the pair potential leads to the formation of a zero-bias peak [6]. Also, a series of experiments in various Josephson-junctions setups in proximity to a two-dimensional InSbAs well are reported in Ref. [7].

\section{The HKP theory}

Hell et al. [1] and Pientka and Keselman et al. [4] studied the properties of the junction (cf. Fig 1) and the conductance through its edge as a function of various

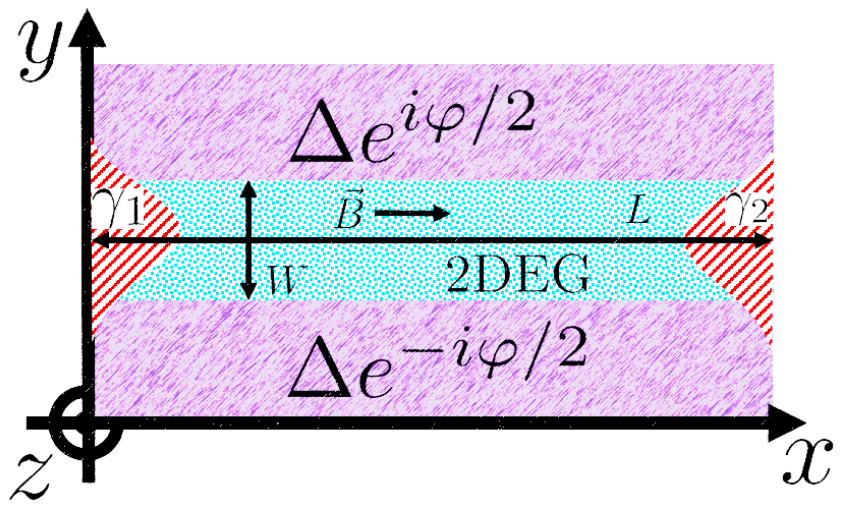

Figure 1: Sketch of the planar Josephson junction. The blue 2D electron gas forms a quasione-dimensional region. The phase difference $\varphi$ between the two superconductors (purple) is controlled by external magnetic flux through a loop connecting them, and in addition a magnetic field $\vec{B}$ applied parallel to the junction (in the $x$ direction) produces a Zeeman splitting. In a topological state, two Majorana zero modes $\left(\gamma_{1}\right.$ and $\left.\gamma_{2}\right)$ appear at the junction's ends. Conductance measurements through the end of the quasi-one-dimensional junction exhibit zero-bias peaks at a certain region of parameters. The measurements are consistent with the predictions of the HKP theory. parameters, among which are the length of the quasi-one-dimensional normal part $L$ and its width $W$, the strength of the spin-orbit coupling, the strength of the normal scattering between the quasi-one-dimensional region and the superconducting pads, and the chemical potential, $\mu$. Most importantly, they studied the phase diagram as a function of the Zeeman energy induced by a magnetic field applied parallel to junction (in the $x$ direction), leading to a spin splitting with the Zeeman energy $E_{\mathrm{Z}}$, and the phase difference between the superconducting pair potentials, $\varphi$.

Assuming that $\mu \gg \Delta$ (the Andreev limit) and a perfect Andreev reflection at the boundaries, HKP find that for $\varphi=0$, the Zeeman energy has to be of the order of the Thouless energy $\left(v_{\mathrm{F}} / W\right.$, with $v_{\mathrm{F}}$ being the Fermi velocity) to stabilize a topological state. However, at $\varphi=\pi$ the Andreev reflection processes are affected in such a way that only a 
very small parallel field that produces a small $E_{\mathrm{Z}}$, is sufficient to drive the system to the topological state. When normal reflections are included and/or $\mu \not \Delta$ the diamond shape of the phase diagram is altered as depicted schematically in Fig. 2.

The main challenge is to find regions in the phase diagram with a large gap at a small magnetic field. Then, the MZM has a short localization length and the superconductor's properties are not degraded due to the magnetic field. Numerical simulations $[3,4]$, in the Andreev limit and in the absence of normal reflections, suggest that such a situation occurs near $\varphi=\pi$. Notably, due to the application of the Zeeman field and finite $\varphi$, there is no symmetry in the value of the gap around $\varphi=\pi$, see Fig. 2. In addition, in this limit HKP find that, in contrast to the wire geometry, which is not necessarily in the Andreev limit and has strong normal reflection at the wire's transversal boundaries, the topological state is not sensitive to the value of $\mu$.

Semi-classical electron trajectories that are parallel to the superconductor give rise to a small gap, as they have a small probability of being Andreev reflected. A zigzag shape for the junction [8] and/or disorder [9] may increase their probability to be Andreev reflected.

The experimental results that I will describe now are in agreement with the theory HKP, and exhibit zero-bias peaks at relatively low magnetic fields. Whether the zero-bias peak is due to an isolated MZM, or rather a result of combinations of MZM, finite $L$ and other modes in the device, requires further study and optimization of the experimental parameters.

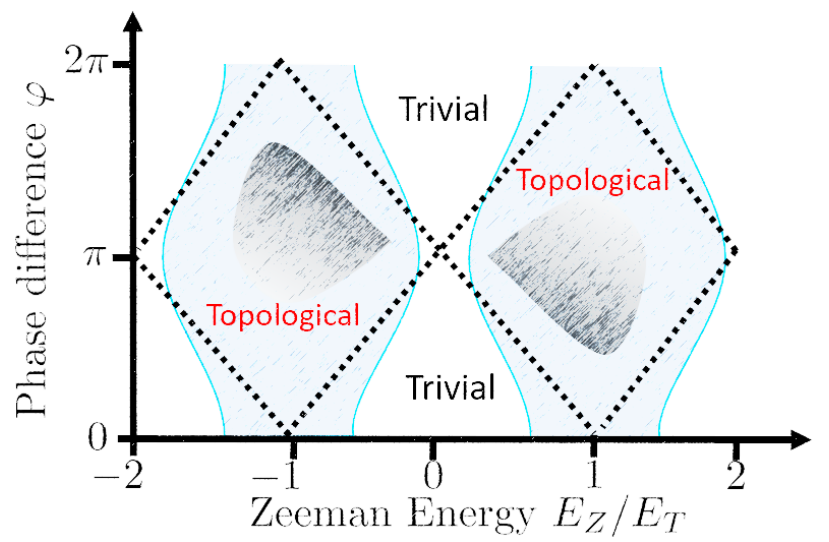

Figure 2: The HKP phase diagram as a function of the Zeeman energy, $E_{\mathrm{Z}}$, in units of $E_{T}=v_{\mathrm{F}} / W$ with $v_{\mathrm{F}}$ being the Fermi velocity and $W$ the width of the normal region. The dashed line denotes the boundary between the topological and trivial phases in the perfect Andreev limit, when the Fermi energy is much larger than the gap, and there is perfect Andreev scattering at the normal-superconductor interface. The shaded area indicates schematically the magnitude of the induced gap in the system, with darker shades corresponding to larger gaps. Notice the $180^{\circ}$ rotation symmetry around $\left(E_{\mathrm{Z}}=0, \varphi=\pi\right)$, which follows from the symmetry of the spectrum under the operation $\vec{B} \rightarrow-\vec{B}$. In particular, there is no symmetry for a fixed $E_{\mathrm{Z}}$ and reflection around $\varphi=\pi$. The blue areas denote the topological regions in the presence of normal reflections and/or when the Fermi energy is not much larger than the superconducting gap.

\section{The Copenhagen and Harvard experiments}

Both experiments at Copenhagen and Harvard investigate a phase-controlled planar Josephson junction. Experimentally it is very different from wire-based devices, and conceptually it is important as there is an additional tuning knob - the phase difference of the superconductors. The experiments include data from different samples under different experimental 
conditions, some of them showing zero-bias peaks while others do not. The experimental papers include a detailed comparison with the theory.

Remarkably, the two experiments are performed on different materials with different growing methods, attesting that the results are universal. At Harvard the planar Josephson junction consists of an 8nm-thick HgTe quantum well contacted by thermally-evaporated aluminium leads about $15 \mathrm{~nm}$ thick. The junction's dimensions are $W \approx 400-600 \mathrm{~nm}$ and $L \approx 1-4 \mu \mathrm{m}$. At Copenhagen the Josephson junctions were fabricated from a planar heterostructure comprising a thin Al layer epitaxially covering a high-mobility InAs twodimensional electron gas (2DEG). Selectively removing an Al stripe of width $W \approx 40-$ $120 \mathrm{~nm}$ and length $L \approx 1.6-5 \mu \mathrm{m}$ defines a normal InAs region, laterally contacted by the superconductor. In contrast to the Harvard experiment where the leads are wider than the coherence length, in the Copenhagen experiment the superconducting leads have a width of order $160 \mathrm{~nm}-1 \mu \mathrm{m}$, which may be smaller than the coherence length of Aluminium.

Both experiments observed that biasing the junction to $\varphi=\pi$ indeed reduces the critical field at which the zero-bias peak appears, with respect to $\varphi=0$. However, the critical field is still of the order of $0.5 \mathrm{~T}$, somewhat smaller than the values obtained in the semiconducting wires devices (without the phase control), and larger than the full-shell experiment [6] at which a zero bias peak is observed at about $0.1 \mathrm{~T}$.

\section{Conclusions}

To summarize, magnetic fields are notoriously known to create quasi-particle states with energy below the superconducting gap, which may lead to severe dephasing phenomena. The experiments that I recommend here are interesting as they show that the manipulations of the superconductors' phases in planar junctions may help in finding the conditions for stabilizing topological states at lower magnetic fields. Future studies, both theoretical and experimental, are needed in order to optimize the conditions for topological superconductivity.

\section{References}

[1] Anton Akhmerov. Search for majoranas: a new piece of the puzzle. Journal club for Condensed Matter Physics, May 2016. doi: 10.36471/JCCM_May_2016_01. URL https: //doi.org/10.36471/JCCM_May_2016_01.

[2] R.M. Lutchyn, E.P.A.M. Bakkers, L.P. Kouwenhoven, P. Krogstrup, C.M. Marcus, and Y. Oreg. Majorana zero modes in superconductor semiconductor heterostructures. Nature Reviews. Materials, 3:52-68, 5 2018. ISSN 2058-8437. doi: 10.1038/s41578-018-0003-1.

[3] Michael Hell, Martin Leijnse, and Karsten Flensberg. Two-Dimensional Platform for Networks of Majorana Bound States. Phys. Rev. Lett, 118(10):107701, Mar 2017. doi: 10.1103/PhysRevLett.118.107701.

[4] Falko Pientka, Anna Keselman, Erez Berg, Amir Yacoby, Ady Stern, and Bertrand I. Halperin. Topological Superconductivity in a Planar Josephson Junction. Physical Review X, 7(2):021032, Apr 2017. doi: 10.1103/PhysRevX.7.021032. 
[5] Alessandro Romito, Jason Alicea, Gil Refael, and Felix von Oppen. Manipulating majorana fermions using supercurrents. Phys. Rev. B, 85:020502, Jan 2012. doi: 10.1103/PhysRevB.85.020502.

[6] S. Vaitiekėnas, M. T. Deng, P. Krogstrup, and C. M. Marcus. Flux-induced Majorana modes in full-shell nanowires. arXiv e-prints, art. arXiv:1809.05513, Sep 2018.

[7] William Mayer, Matthieu C. Dartiailh, Joseph Yuan, Kaushini S. Wickramasinghe, Alex Matos-Abiague, Igor Žutić, and Javad Shabani. Phase signature of topological transition in Josephson Junctions. arXiv e-prints, art. arXiv:1906.01179, Jun 2019.

[8] Tom Laeven, Bas Nijholt, Michael Wimmer, and Anton R. Akhmerov. Enhanced proximity effect in zigzag-shaped Majorana Josephson junctions. arXiv e-prints, art. arXiv:1903.06168, Mar 2019.

[9] Arbel Haim and Ady Stern. Benefits of Weak Disorder in One-Dimensional Topological Superconductors. Phys. Rev. Lett, 122(12):126801, Mar 2019. doi: 10.1103/PhysRevLett. 122.126801 . 